\title{
DYNAMIC CHARACTERISTICS OF LOW-PRESSURE WATER HYDRAULIC SYSTEM
}

\author{
Yoshihiro Yata ${ }^{*}$ Takeshi Nakada $^{*}$ Yasuo Sakurai $^{* *}$ Kazuhiro Tanaka ${ }^{* * *}$ \\ * Tokyo Denki University, \\ 2-1200, Muzai gakuenndai, Innzai City , Chiba, 270-1382 Japan \\ (E-mail:nakada@sie.dendai.ac.jp) \\ * * Ashikaga Institute of Technology, \\ 268-1, Oomaecho, Ashikaga City, Tochigi, 326-8558 Japan \\ $* * *$ Kyusyu Institute of Technology, \\ 680-4, Oojikawazu, Iizuka City, Fukuoka, 820-8502 Japan
}

\begin{abstract}
Low-pressure water hydraulics is an attractive technology having the low price of pneumatics, good controllability and stiffness of oil hydraulics as well as safety environment. This paper deals with the dynamic characteristics of the low-pressure water hydraulic system consisting of a vortex pump, a flexible tube with a length of $10 \mathrm{~m}$, an electro-magnetic on-off valve and a single rod cylinder with some inertia loads. The step responses on the pressure and the piston displacement in the water hydraulic system under the operational conditions of a low pressure are obtained experimentally. The computer simulation of the system under the above conditions was done by using the simulation software of OHC-Sim developed by our research group. The efficiency in the system is also discussed on the basis of the system simulation.
\end{abstract}

\section{KEY WORDS}

Water hydraulic drive system, Dynamic characteristics, Low pressure supply

\section{NOMENCLATURE}

$C$ : Wave velocity of water $[\mathrm{m} / \mathrm{s}]$

$K$ : Bulk modulus [MPa]

$K_{e}: \quad$ Equivalent bulk modulus of tap water in flexible tube $[\mathrm{MPa}]$

$M$ : Load mass $[\mathrm{kg}]$

$P_{S}: \quad$ System pressure $[\mathrm{MPa}]$

$P_{1}: \quad$ Pressure on piston side $[\mathrm{MPa}]$

$P_{2}: \quad$ Pressure on rod side $[\mathrm{MPa}]$

$X$ : Piston displacement $[\mathrm{mm}]$

$$
\begin{array}{rll}
\rho: & \text { Density }\left[\mathrm{kg} / \mathrm{m}^{3}\right] \\
\nu: & \text { Kinematic viscosity }\left[\mathrm{m}^{2} / \mathrm{s}\right]
\end{array}
$$

\section{INTRODUCTION}

The oil hydraulic systems have been widely used in many fields of the industries because of their good performances and fast responses. However, it is of overriding importance today that we settle the environmental problem and the problem of recycling the 
natural resources. To solve these problems latent in the oil hydraulics, the researches and developments of the water hydraulic systems make progress in many countries. There are still plenty of problems lying in realizing the water hydraulic systems, which can be supplied to the industrial markets. The main problems [1] are as follows:

(1) Obtaining the knowledge for dynamic characteristics of water hydraulic systems.

(2) Selection of suitable construction materials against generation of rust,

(3) Quality control of water.

In this study we firstly construct a water hydraulic system similar to a basic oil hydraulic system. The system operates normally, and the dynamic characteristics of the system are made clear in the experiment. Secondly, the dynamic characteristics of the system are analyzed in the computer simulation. The computer simulation results are evaluated by the experimental results. The simulation results show almost the same tendency as the results of the experiment, though the both results are slightly different in quantity. The superiority of the water hydraulics over the oil hydraulics is recognized from the simulation result.

\section{CONSTRUCTION OF WATER HYDRAULIC SYSTEM}

The schematic diagram of a water hydraulic system used in the experiment is shown in Figure 1. The system consists of a vortex pump, a relief valve, an electro-magnetic on-off solenoid valve and a single rod cylinder. The on-off solenoid valve is connected to the vortex pump by a flexible tube with a length of $10 \mathrm{~m}$.In this study since we construct a water hydraulic system, aiming at the application to a nursing care system, the single rod cylinder is set up vertically, and its load is the inertia load equivalent to a weight of a person. It is considered that the water hydraulic system operated by a low pressure on the order of a tap water pressure has the highest adaptability to the various kinds of the industrial markets under the current state of affairs. The supply pressure in this system is restricted to be lower than $0.5 \mathrm{MPa}$ in the experiment. So, pneumatic elements are applied to all the elements in the system, except for the vortex pump and the relief valve in it. The specification of the main elements used in the system is as follows:

- Working fluid

- tap water

- Vortex pump

- Maximum pressure $P_{s}<0.5 \mathrm{MPa}$

- Maximum flow rate $Q<801 / \mathrm{min}$

- Solenoid valve

- Diameter of spool $=13.7 \times 10^{-3} \mathrm{~m}$
- Flexible tube between the solenoid valve and the vortex pump

- Length $=10 \mathrm{~m}$

- Diameter $=1 \times 10^{-2} \mathrm{~m}$

- Steel pipe between the solenoid valve and the cylinder

- Length $=0.5 \mathrm{~m}$

- Diameter $=1 \times 10^{-2} \mathrm{~m}$

- Cylinder

- Cross sectional area of piston $=1.96 \times 10^{-3} \mathrm{~m}^{2}$

- Cross sectional area of rod $=1.65 \times 10^{-3} \mathrm{~m}^{2}$

- Maximum piston stroke $=25 \times 10^{-2} \mathrm{~m}$

- Inertia load mass: $10 \sim 50 \mathrm{Kg}$

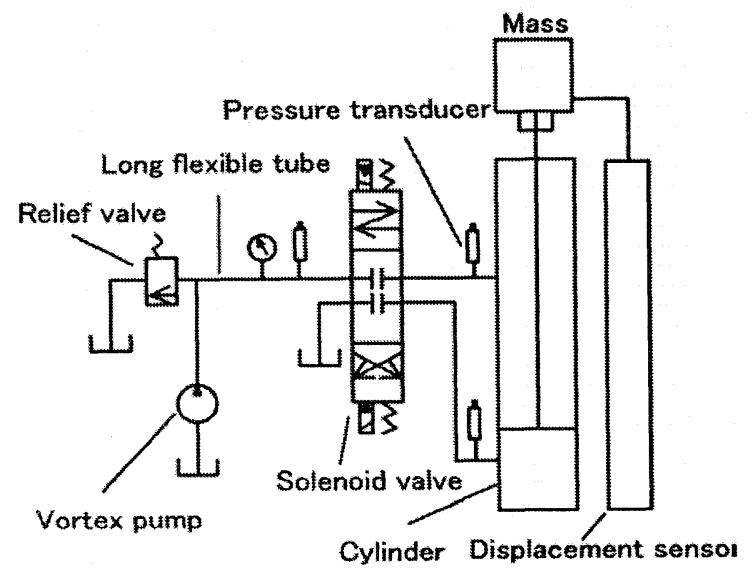

Figure 1 Water hydraulic system

\section{EXPERIMENT AND COMPUTER SIMULATION}

\subsection{EXPERIMENT}

In order to make clear the dynamic characteristics of the system, we measure $P_{S}$ the inlet pressure (system pressure) of the solenoid valve, $P_{1}$ the pressure on the piston side and $P_{2}$ on the rod side, and $X$ the piston displacement. Each pressure is measured with each pressure transducer and the piston displacement with an inductance type displacement sensor. In the initial state in the experiment the position of the piston is set to the bottom of the cylinder, and the solenoid valve is closed. The outlet pressure of the vortex pump is adjusted to be constant by the relief valve. The dynamic characteristics on $P_{S}, P_{1}, P_{2}$ and $X$ are measured in the experiment when the input of the step function is given to the solenoid valve.

\subsection{COMPUTER SIMULATION}

We use a simulation package OHC-Sim [2] to analyze the water hydraulic system in the computer simulation, which has been developed by our research group with support of JFPS. OHC-Sim has a user-friendly graphical interface in Windows environment, and provides easy design for an oil-hydraulic circuit. 


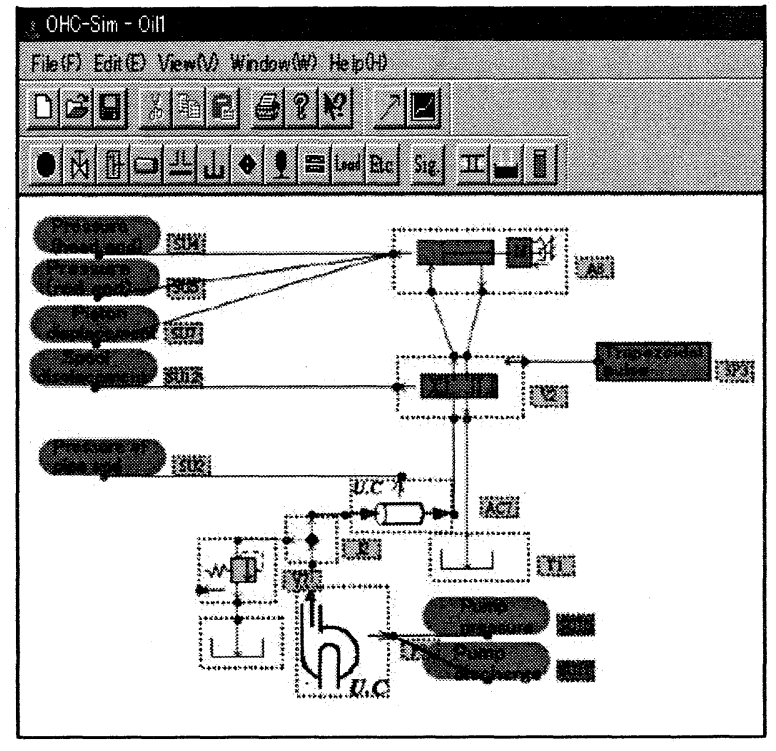

Figure 2 Construction of simulation circuit by OHC-Sim

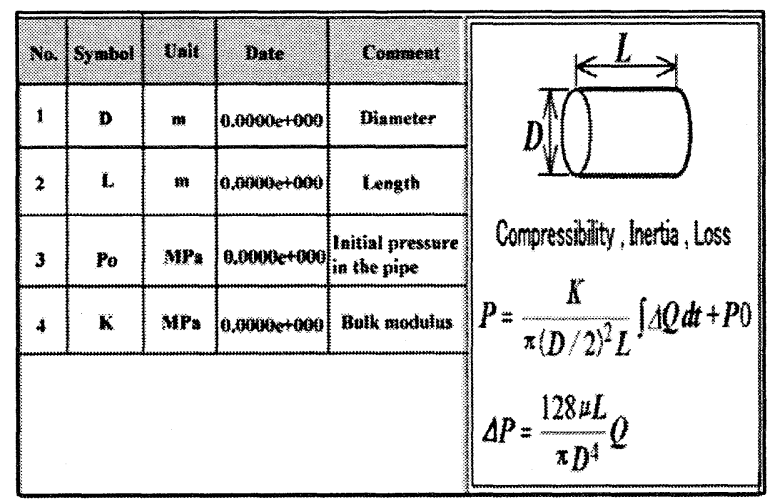

Figure 3 Dialog box for setting parameter (tube)
At present OHC-Sim is provided only in Japanese Version, but an English Version will be arranged in the near future. The models for typical hydraulic components were registered in it, and users can register the models for their own new hydraulic components to the database, if necessary. Figure 2 shows the construction of the system in this study by OHC-Sim.

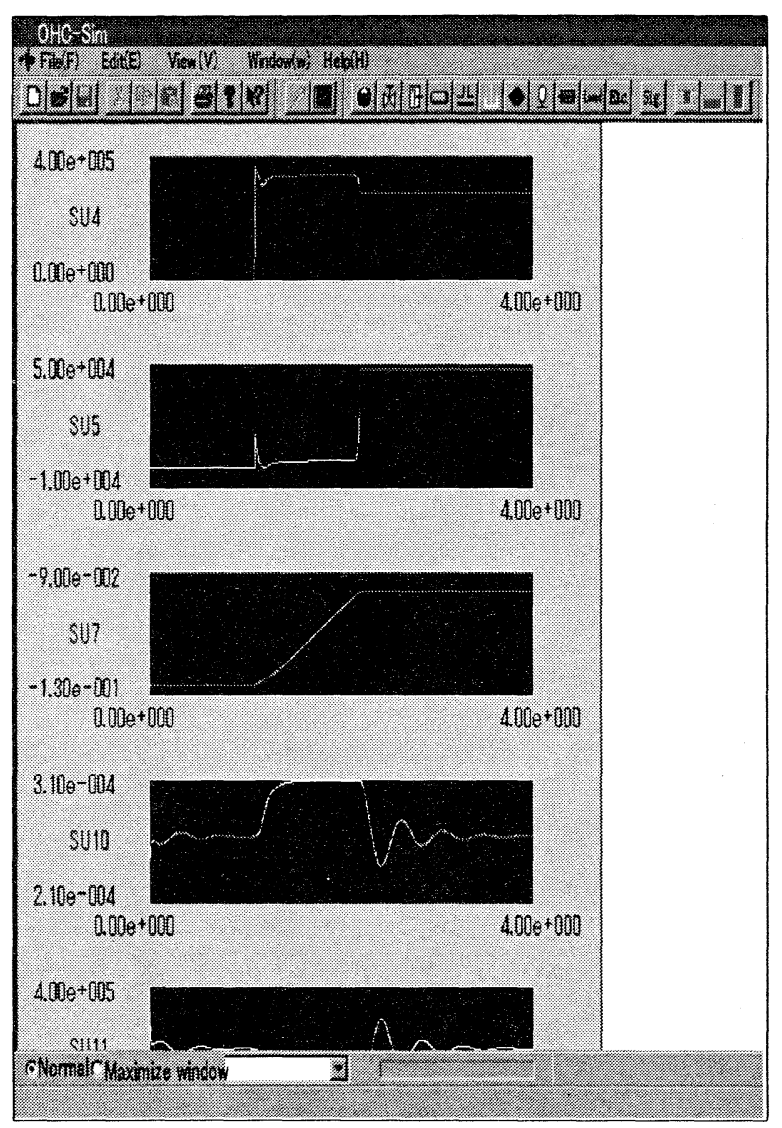

Figure 5 Display of simulation result

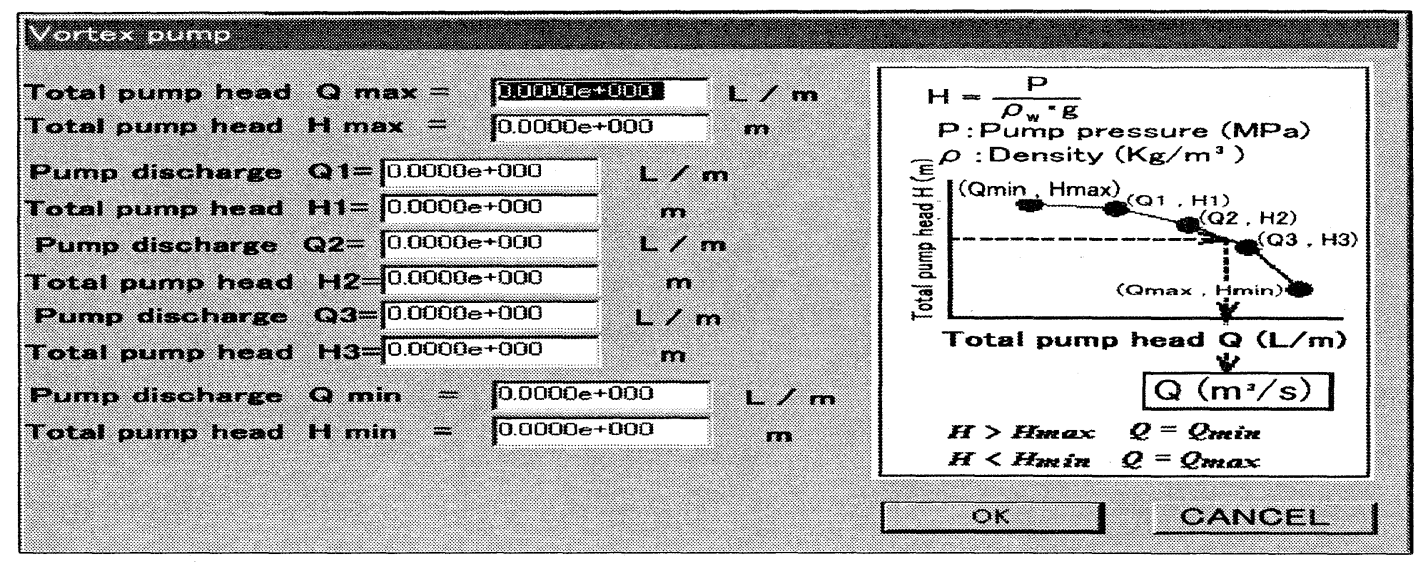

Figure 4 Dialog box for setting parameter (vortex pump) 
The Dialog Boxes to set the parameters on the tube, vortex pump are shown in Figure 3 and Figure 4. Using these Dialog Boxes sets the necessary parameters, and the calculation is carried out. Thus we can obtain the simulation results, respectively. Figure 5 shows an example of a display of simulation results. Firstly, the experiment and the computer simulation were conducted on the system equipped a very short steel pipe instead of the long flexible tube shown in Figure 1 to confirm the authenticity of the simulation by OHC-Sim in advance. The experimental result in case of initial system pressure of $0.4 \mathrm{MPa}$ and the load mass of $50 \mathrm{~kg}$ and the corresponding simulation result are shown in Figure 6 and Figure 7, respectively. It is clarified from Figure 6 and Figure 7 that the simulation results show almost the same tendency as the results of the experiment in the responses of $P s$ the system pressure, $P_{1}$ the pressure on the piston side and $P_{2}$ the pressure on the rod side. However we want to know about characteristics of water hydraulic system in the simulation, it can be said that OHC-Sim is effective on the simulation of the dynamic characteristics of in case of the water hydraulic system with the short steel pipe.

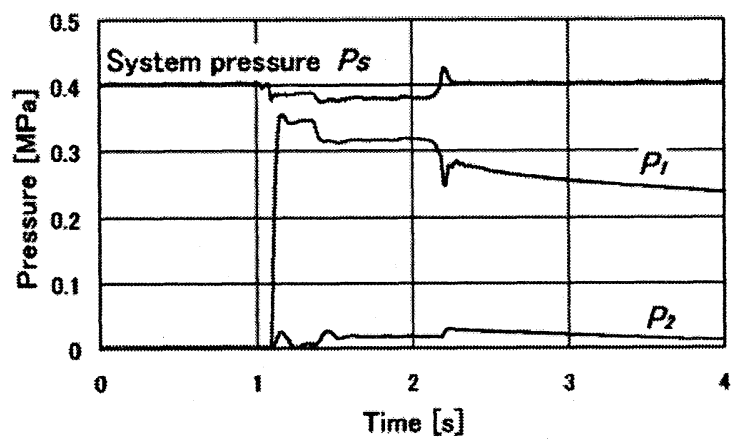

Figure 6 Pressure response of water hydraulic system with very short steel pipe in experiment (Load mass: $50 \mathrm{~kg}$ )

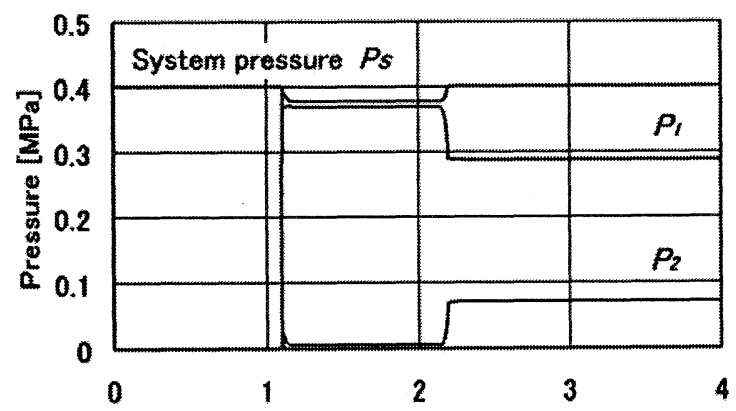

Figure 7 Pressure response of water hydraulic system with very short steel pipe in simulation (Load mass: $50 \mathrm{~kg}$ )

\section{EXPERIMENTAL RESULT AND SIMULATION RESULT [3][4] [5]}

The experiment and the simulation on the system with the long flexible tube shown in Figure 1 were done under the various conditions such as the system pressures of $0.3 \mathrm{MPa}$ and $0.4 \mathrm{MPa}$ and the load masses of $10,20,30,40$ and $50 \mathrm{~kg}$. Figure 8 shows the experimental result in case of the system pressure of 0.4 $\mathrm{MPa}$ and the load mass of $50 \mathrm{~kg}$. In Figure 8 the solenoid valve is opened stepwise at a time of 1 second and closed at a time of 2 seconds. The remarkable damped oscillation on the system pressure $P_{S}$ is appeared after a time of 2 seconds in Figure 8 . The frequency of the oscillation is $2 \mathrm{~Hz}$ and the maximum pressure generated reaches to about 1.8 times as large as the initial system pressure of $P_{S}$.

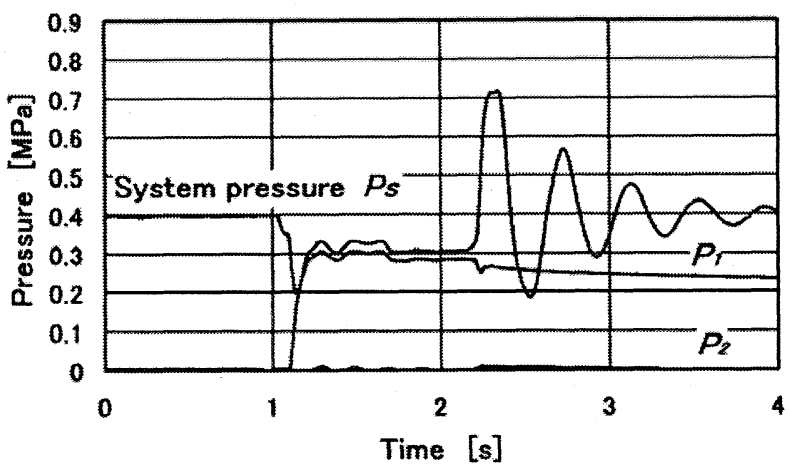

Figure 8 Pressure response of water hydraulic system in experiment (Load mass: $50 \mathrm{~kg}$ )

Table 1 Typical fluids properties (temperature:20 ${ }^{\circ} \mathrm{C}$ )

\begin{tabular}{|c|c|c|}
\hline & Tap water & Oil \\
\hline$\rho\left(\mathrm{kg} / \mathrm{m}^{3}\right)$ & 1000 & 880 \\
\hline$\nu\left(\mathrm{m}^{2} / \mathrm{s}\right)$ & $1.0 \times 10^{-6}$ & $34.0 \times 10^{-6}$ \\
\hline$K(\mathrm{GPa})$ & 2.4 & 1.5 \\
\hline
\end{tabular}

Since the experiment was done at the temperature of $20^{\circ} \mathrm{C}$, some conventional values at the same temperature for the important parameters of the tap water and the oil are given in Table 1 [6] to conduct the computer simulation of the dynamic characteristics of the water hydraulic system and the oil hydraulic system. In the experiment the long flexible tube is used, and it is considered that the value of $K$ the bulk modulus of the tap water in it is largely different from the value in Table 1. Therefore $K_{e}$ the equivalent bulk modulus of the tap water in the flexible tube is obtained in the experiment. 
Since we get the very small value of $2 \mathrm{MPa}$ as the value of $K_{e}$, this value is used on the flexible tube in the simulation. The simulation model for the flexible tube is given by the bi-block lumped parameter system, and the laminar flow.

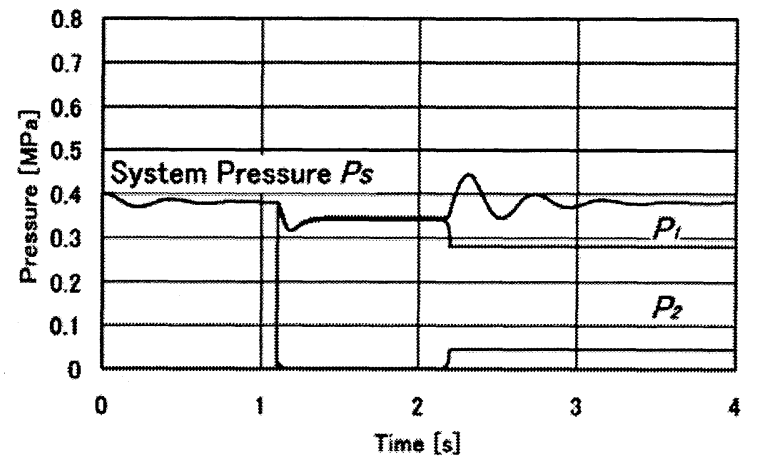

Figure 9 Pressure response of water hydraulic system in simulation (Load mass: $50 \mathrm{~kg}$ )

Figure 9 shows the simulation results of this system under the same operation conditions as the experiment. The frequency of the oscillation on the system pressure in the simulation is about $2 \mathrm{~Hz}$, and it coincides well with the frequency of the oscillation appeared in the experiment. However, the large difference is recognized at the point of the magnitude of the oscillation. Since it is considered that the striking difference is caused by the inaccuracy of the model for the flexible tube such as the rough subdivision and the laminar flow, the revise of the current model in OHC-Sim is now under consideration.

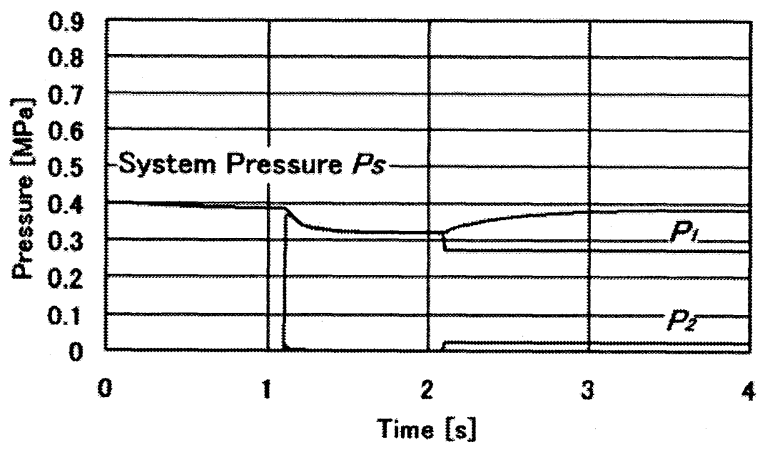

Figure 10 Pressure response of oil hydraulic system in simulation (Load mass: $50 \mathrm{~kg}$ )

Figure 10 shows the simulation result corresponding to Figure 9 when the oil is used in place of the water as the working fluid. In the simulation the value of $K_{e}$ the equivalent bulk modulus of the tap water is adopted as the bulk modulus of the oil in the flexible tube, because the elastic behavior of the flexible tube has an effect largely on the bulk modulus of the fluid in it. The oscillation of the system pressure disappears in Figure 10 , since the viscosity of the oil is about 34 times as large as that of the water. It is more important in the water hydraulics than in the oil hydraulics that the consideration should be paid into such pressure oscillation generated in the system.

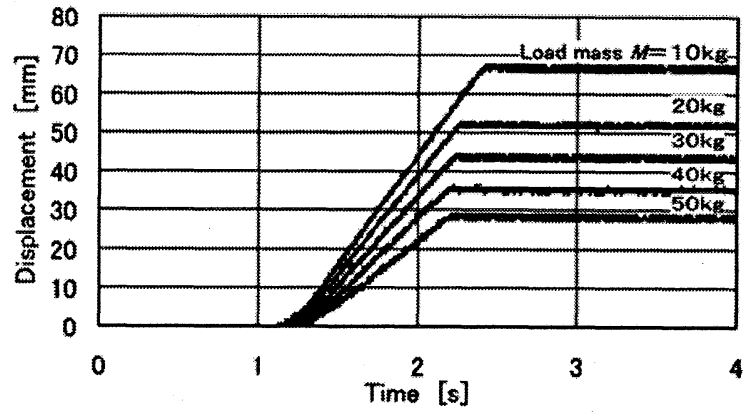

Figure 11 Response of piston traveling in water hydraulic system in experiment (Initial system pressure: $0.4 \mathrm{MPa}$ )

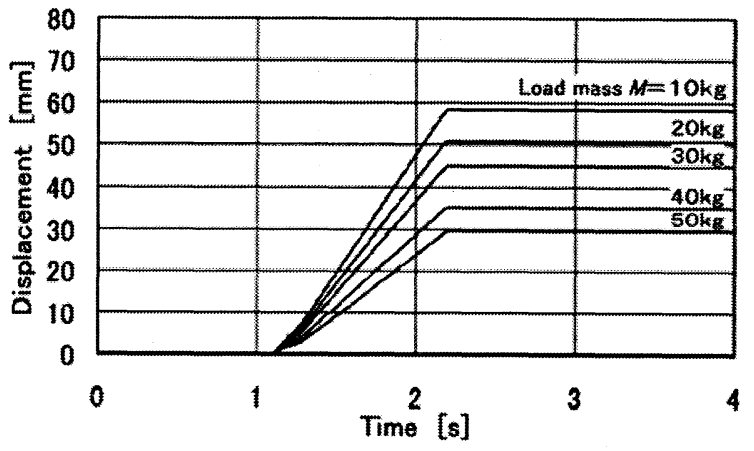

Figure 12 Response of piston traveling in water hydraulic system in simulation (Initial system pressure: $0.4 \mathrm{MPa}$ )

Figure 11 and Figure 12 show the experimental results of the piston displacement $X$ and the corresponding simulation result, respectively. It is confirmed in the experiment that the smooth traveling of the piston can be expected even in the low-pressure water hydraulic system. The both results shown in Figure 11 and Figure 12 show almost the same characteristics in quality and in quantity expect for case of $M=10 \mathrm{~kg}$.

Figure 13 shows the simulation result of the piston displacement, when the oil is used in place of the water as the working fluid. The piston displacement in case of the oil amounts to $70 \%$ as small as that in case of the water. It is considered that this reduction results from the increase of the pressure loss in the long flexible tube, 
since the viscosity of the oil is much larger than that of the water.

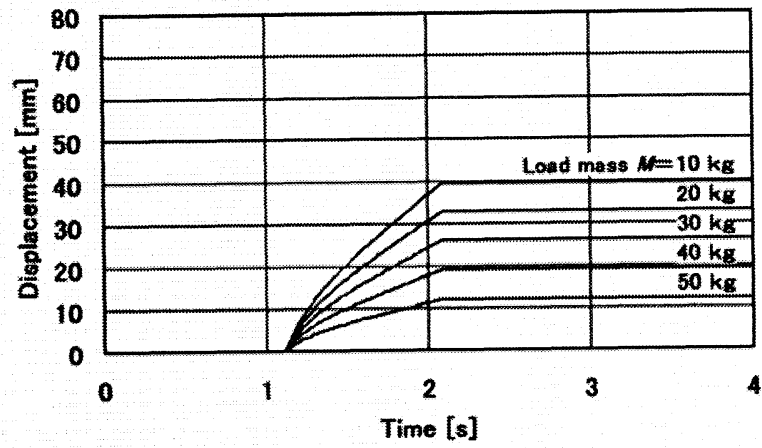

Figure 13 Response of piston traveling in oil hydraulic system in simulation (Initial system pressure: $0.4 \mathrm{MPa}$ )

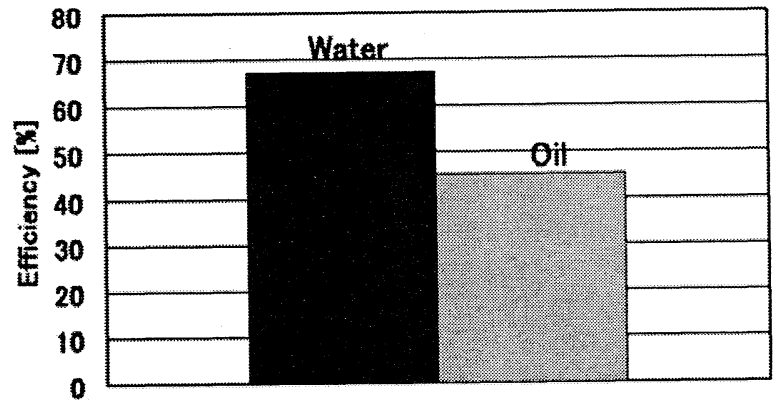

Figure 14 Comparison of the efficiency between water hydraulics and oil hydraulics (Initial system pressure: $0.4 \mathrm{MPa}$ ) (Load mass: $50 \mathrm{~kg}$ )

In order to investigate the energy saving between the water hydraulics and the oil hydraulics, the efficiency in each system is obtained in the simulation. The efficiency means the ratio of the kinetic energy consumed at the cylinder to the energy of the fluid flowing into the inlet of the flexible tube while the piston travels upwards $30 \mathrm{~mm}$ form the bottom of the cylinder. The simulation result is shown in Figure 14 and the water hydraulics is superior to the oil hydraulics at the point of the energy saving.

\section{CONCLUSIONS}

The water hydraulics is very attractive technology benign to the human being and the earth environment. The basic characteristics of the low-pressure water hydraulic system with a long flexible tube in it are made clear in the experiment and the computer simulation. Though the pneumatic components are used in place of the water hydraulic components to construct the system in the experiment, the system can be gotten to work properly. Since the more noticeable tendency of generating the pressure surge is recognized in the water hydraulic system than in the oil hydraulic system, the sufficient consideration of the pressure surge should be taken into account in designing the water hydraulic system. The water hydraulic system is superior to the oil hydraulic system at the point of the energy saving and the resource saving.

\section{REFERENCES}

1. Shinpei Miyakawa, et al. , Report of Research Committee on Water Hydraulic Drive System, THE JAPAN FLUID POWER SYSTEM Society, 2000

2. Yasuo Sakurai, et al. , Introduction of Innovative Program OHC-Sim for Oil Hydraulic Circuit Simulation, Third JHPS International Symposium on Fluid Power, 1996, pp.679-682

3. Yoshihiro Yata, et al. , Simulation Dynamic Characteristic of Water Hydraulic System by OHC-Sim , JSME Annual Meeting , 2001, pp.299-300

4. Yoshihiro Yata, et al ., Analysis of Low Pressure Water Hydraulic Drive System with Flexible Long Tube, JSME Annual Meeting, 2002. pp.163-164

5. Jussi Aaltonen, et al ., Experiment on the Low Pressure Water Hydraulic Systems, Forth JHPS International Symposium on Fluid Power , 1999, pp.357-363

6. Kazusi Sanada , A Study on Control Performance of Water Hydraulic Systems, Forth JHPS International Symposium on Fluid Power , 1999, pp 721-726 